Mappemonde

Revue trimestrielle sur l'image géographique et les formes du territoire

119 | 2017

Varia

\title{
Donner à voir la valorisation des déchets urbains par la microgéographie des « lieux de l'ordure »
}

Adeline Pierrat

OpenEdition

Journals

Édition électronique

URL : http://journals.openedition.org/mappemonde/2424

DOI : $10.4000 /$ mappemonde.2424

ISSN : 1769-7298

Éditeur

UMR ESPACE

Référence électronique

Adeline Pierrat, « Donner à voir la valorisation des déchets urbains par la microgéographie des « lieux de l'ordure » », Mappemonde [En ligne], 119 | 2017, mis en ligne le 01 janvier 2017, consulté le 14 septembre 2020. URL : http://journals.openedition.org/mappemonde/2424

Ce document a été généré automatiquement le 14 septembre 2020

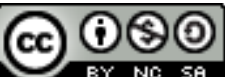

La revue Mappemonde est mise à disposition selon les termes de la Licence Creative Commons Attribution - Pas d'Utilisation Commerciale - Partage dans les Mêmes Conditions 4.0 International. 


\title{
Donner à voir la valorisation des déchets urbains par la microgéographie des « lieux de l'ordure »
}

\author{
Adeline Pierrat
}

\author{
Référence de la thèse \\ PIERRAT A. (2014). Les lieux de l'ordure de Dakar et d'Addis Abäba. Territoires \\ urbains et valorisation non institutionnelle des déchets dans deux capitales \\ africaines. Thèse de doctorat de géographie, Paris, Université Paris 1 Panthéon- \\ Sorbonne, $559 \mathrm{p}$.
}

1 Les déchets sont le fléau des villes d'Afrique. Le processus qui redonne de la valeur aux déchets, tout en contribuant à mieux les gérer, est appelé «valorisation». Transformant les ordures de nuisances en ressources, celui-ci est logiquement de plus en plus mis en avant. Il est l'objet de notre réflexion et sa dimension spatiale est notre sujet de recherche. En traitant des cas de Dakar, capitale du Sénégal et d'Addis Abäba, capitale de l'Éthiopie, notre thèse a pour ambition de comprendre, à travers leur valorisation non institutionnelle, comment les déchets créent de nouveaux territoires et interfèrent avec les transformations urbaines guidées par les paradigmes du " propre » et du « vert».

2 La cartographie à grande échelle est l'outil que nous avons privilégié pour donner à voir et pour étudier des quartiers réduits à l'invisibilité par les politiques du propre et du vert.

3 L'iconographie proposée témoigne d'une organisation de l'espace par le secteur informel. Pour d'autres catégories de populations, les circulations et les réseaux de Produits Issus de la Valorisation des Déchets (PIVD) font de certains lieux marginaux des centres et, généralement, donnent à voir d'autres pratiques de la ville, notamment celles de businessmen des décharges et des plus démunis. 
Ce travail propose une géographie de la valorisation des déchets qui repose sur l'identification, la localisation, l'examen et la mise en relation de ce que nous appelons les «lieux de l'ordure " (décharges, marchés des déchets, etc.). En conjuguant les éléments de définition des hauts lieux proposés par André Micoud (1991), nous considérons les «lieux de l'ordure $»^{1}$ comme des hauts lieux de la valorisation, caractérisés par une « haute valeur ajoutée " (Brochot et de la Soudière, 2010, p. 10), dans un espace « circonscrit» (Debarbieux, 1995, p. 107).

5 Les enquêtes de terrain qualitatives (questionnaires et entretiens) et les cartes proposées ici, sont tirées du cas additien.

\section{Cartographier des espaces de l'informel pour rendre visibles des processus}

6 La valorisation des déchets en Afrique est principalement aux mains du secteur informel. Pour examiner les différentes étapes de ce processus, tout en éludant la difficulté de travailler sur le secteur informel qui présente des contours sociaux et spatiaux flous, ma démarche consiste à élaborer la démonstration à partir des acteurs et des activités de valorisation.

7 Ils sont localisés et dans notre étude ils sont soit considérés comme marginaux - c'est le cas des sites de décharges non contrôlés, montagnes d'ordures et dépotoirs à ciel ouvert, situés en périphérie des agglomérations - soit il s'agit d'espaces interstitiels, non fonctionnels, de l'espace urbain où se concentrent les activités informelles. Dans les deux cas, ils marquent une rupture dans le paysage urbain, mais n'ont jamais été cartographiés. Or, ma thèse s'appuie sur l'hypothèse que la cartographie à grande échelle révèle la construction de territoires qui résulte des activités de valorisation: fouille et récupération des déchets, tri, transformation, revente, etc. Les deux images choisies illustrent la démarche adoptée et sont également des résultats.

8 La figure 1 examine comment le site de décharge de Koshe Repi ne remplit pas seulement un rôle de dépôt des ordures. La figure 2 illustre une démarche semblable élaborée à partir des étapes qui suivent la fouille et le tri des ordures et qui se concentrent dans des lieux que je qualifie de «marchés ». Ici nous traitons du cas de Shint Tära, qui signifie littéralement « quartier des boues ».

Réaliser les plans des espaces interstitiels, souvent cachés par des murs ou autres éléments urbanistiques, nécessite de longs repérages et le recours à l'observation participante. Il a d'abord fallu créer les fonds, à partir d'une photographie aérienne qui sert de référence dans le cas du site de décharge. La rue principale qui jouxte le quartier commerçant de Menalesh Tära a, quant à elle, servi de point de repère pour comprendre le fonctionnement interne du marché des déchets. Des entretiens et des questionnaires ont permis d'afFiner les plans et de confronter les données issues de l'observation aux pratiques spatiales des acteurs informels. 


\section{Du non-lieu au lieu : le plan au cœur de la démonstration}

10 Partant d'un espace qui s'apparente à un no man's land détritique, la figure 1 donne à
voir comment les décharges sont également des lieux de travail, de vie, de commerce
créés par la récupération des déchets et approprié par le secteur informel.

Koshe Repi fait office de rupture dans une partie du sud-ouest de la ville, à $6 \mathrm{~km}$ du centre, où l'habitat prédomine. Considérée comme une irrégularité paysagère, elle peut aussi représenter une limite physique, associée à la Ring Road (boulevard circulaire), frontière séparant les quartiers d'habitat moderne (au sud) des quartiers populaires (au nord). Si l'on considère les activités que la décharge supporte, une autre interprétation est possible: le site apparaît comme un pôle d'activités organisées. La partie ancienne correspond à une accumulation de déchets verts, ce qui explique et légitime la présence du planteur de tèf (céréale locale). Les récupérateurs (ou chiffonniers) et les grossistes sont installés autour de la plateforme de dépôt des ordures fraîches qui viennent d'être collectées en ville et migrent généralement avec elle. La spécialisation dans le ramassage des herbes séchées s'efFectue logiquement dans les zones de dépôt d'ordures un peu moins récentes. L'emplacement des zones de stockage des matériaux triés apparaît sans surprise à proximité de la route asphaltée. À l'est de l'entrée principale de Koshe Repi, le squat des jeunes hommes constitue un repère pour les récupérateurs qui viennent s'abriter du soleil et discuter. Les acheteurs et revendeurs sont installés autour de la plateforme. La chronologie de la mise en décharge explique l'organisation spatiale de la récupération des ordures. Cette carte révèle enfin un territoire, avec ses limites, son appropriation par un type d'acteurs dont le jeu implique une réflexion sur le pouvoir et le foncier. Ce territoire convoité attire de nombreux migrants qui en font ensuite le lieu de leur vie quotidienne. 
Figure 1. Vue aérienne (en bas) et cartographie (en haut) de la décharge de Koshe Repi à Addis Abäba

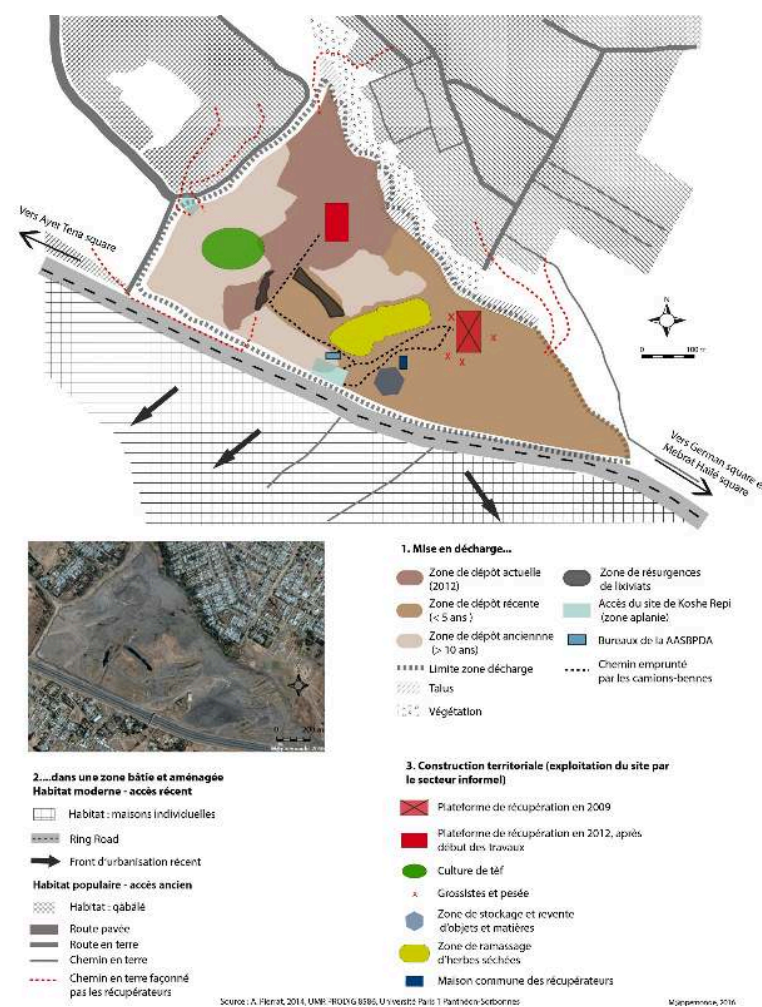

Source : A. Pierrat, 2014, UMR PRODIG 8586, Université Paris 1 Panthéon Sorbonne

12 La seconde iconographie choisie se rapporte à la transformation et la commercialisation des Produits Issus de la Valorisation des Déchets (PIVD). 
Figure 2. Le quartier de Shint Tära à Addis Abäba : marché des déchets

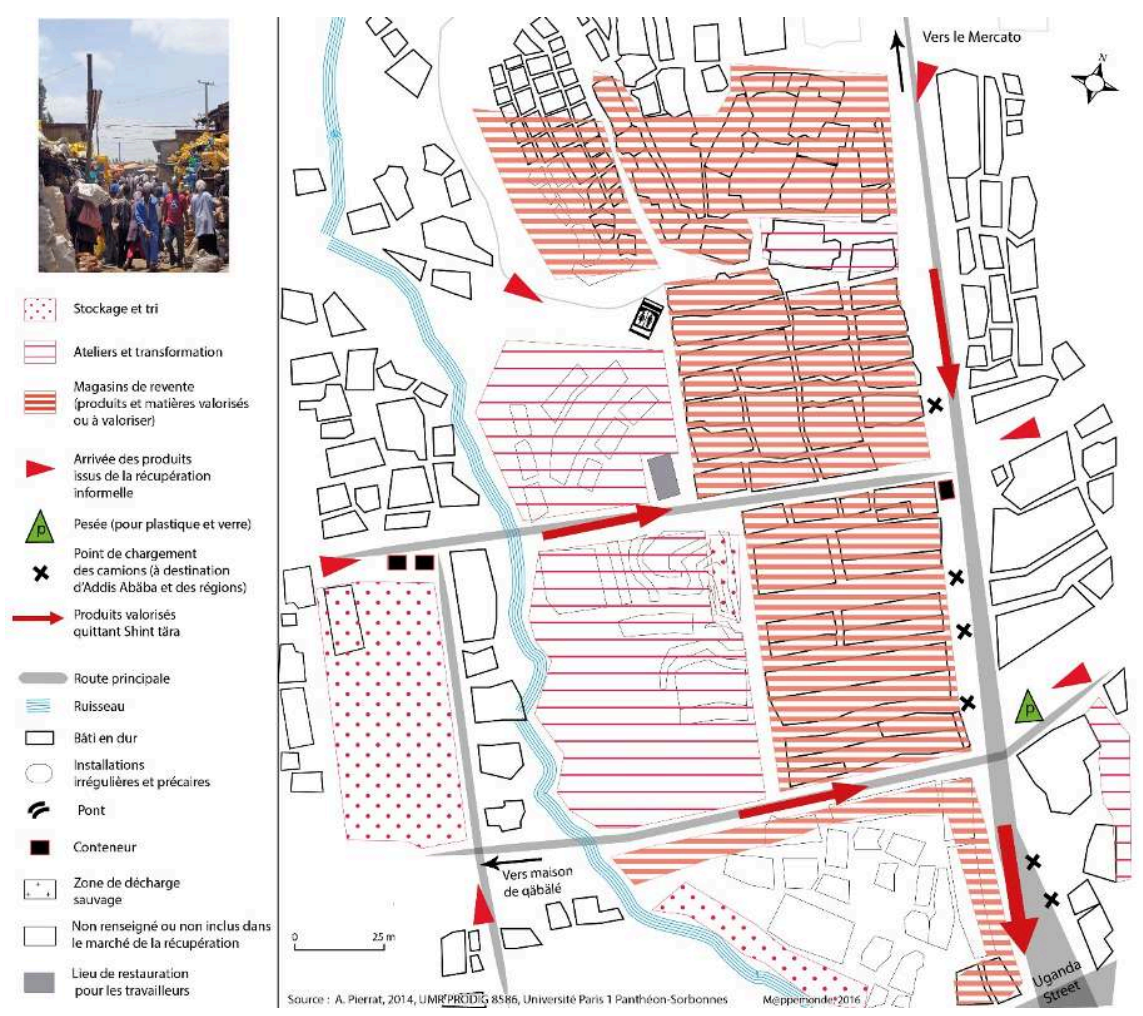

Source : A. Pierrat, 2014, UMR PRODIG 8586, Université Paris 1 Panthéon Sorbonne

13 Sur la photographie (à gauche), les bidons accrochés signalent le changement de quartier, les fers à béton font office de rupture entre la rue, espace public, et le marché de la récupération, domaine de l'informel. La figure 2 s'organise à partir de la route principale desservie par des camions de transport de marchandises. On peut ensuite observer une hiérarchie au sein du réseau depuis la route en asphalte jusqu'aux secteurs les plus éloignés de cet axe. Les voies sont d'abord accessibles en charrette ou en voiture individuelle, puis uniquement à pied. Les figurés " points » et " hachures » correspondent aux différentes étapes de la valorisation (tri, transformation, revente). L'organisation spatiale du marché de Shint Tära repose sur un double découpage, selon les étapes de la valorisation et selon les matériaux recyclés. Le long des ruelles pavées qui composent la partie irrégulière du marché, les ateliers sont regroupés par produits valorisés. Facilement repérables sur le plan, ils marquent une rupture avec les boutiques les plus anciennes construites en dur et qui suivent un plan géométrique, à l'instar des blocs qui composent le Mercato voisin (marché de gros d'Addis Abäba). Cette carte est à comparer à celles d'autres marchés que nous avons pu réaliser à Dakar (Pierrat, 2014).

D'une part, ces dispositifs cartographiques résultant de la microgéographie permettent de reconsidérer les fonctions de certains lieux (figure 1) et, d'autre part, cette démarche permet de révéler des lieux de la ville (figure 2) tout en décryptant leur organisation interne. Ces deux plans sont représentatifs de mes résultats: les sites de décharges sont aussi des lieux stratégiques du fonctionnement de la ville. Ils sont des lieux de survie, d'habitation et de travail pour près de 500 récupérateurs. Les marchés des déchets abritent de nombreux ateliers qui occupent environ 2000 artisans et vendeurs, attirent des clients de tout le pays et permettent l'accès à la consommation 
des ménages pauvres pour qui ces lieux font partie intégrante de leurs pratiques spatiales. Mais cette échelle de travail illustre également le franchissement des frontières intra-urbaines entre les marges et les autres espaces de l'agglomération, dans des contextes africains où le modèle visé est celui de la ville-vitrine (Gervais Lambony, 2003).

\section{BIBLIOGRAPHIE}

BRochot A., DE LA SOUdière M. (2010). « Pourquoi le lieu ? ». Communications, vol. 2, n 87, p. 5-16. DEBARBIEUX B. (1995). « Le lieu, le territoire et trois figures de rhétorique ». Espace géographique, vol. $24, \mathrm{n}^{\circ} 2$, p. $97-112$.

GERVAIS LAMBONY Ph. (2003). Territoires citadins. Quatre villes africaines. Paris : Belin coll. « Mappemonde », 271 p. ISBN 978-2-7011-3375-1

MICOUD A. (1991). Des Hauts Lieux : la construction sociale de l'exemplarité. Paris : Éditions du CNRS, 133 p. ISBN 2-222-04589-4

NORA P. (1984). Les lieux de mémoire. Tome I. «La république ». Paris : Gallimard, 720 p. ISBN 2070701921

\section{NOTES}

1. En référence aux « lieux de mémoire » de Pierre Nora (Nora, 1984).

\section{INDEX}

Mots-clés : déchets, informel, prix de thèse 2015, territorialités, valorisation

\section{AUTEUR}

\section{ADELINE PIERRAT}

Docteure en géographie. Chercheuse associée à l'UMR 8586 Prodig. Chargée de projets

"Assainissement, Déchets" au Gret

pierrat[at]gret.org 